\title{
LUCAS FERNÁNDEZ NAVARRO. EL PRIMER GEÓLOGO ESPAÑOL QUE PISÓ LAS ISLAS CHAFARINAS
}

\author{
José Luis Barrera Morate \\ Geólogo \\ Miembro de la International Commission on the \\ History of Geological Sciences (INHIGEO)
}

Resumen: El geólogo Lucas Fernández Navarro fue el primer vulcanólogo español. Su carrera científica y académica en el Museo de Ciencias y en la Universidad Central (Madrid) le llevó a ser una figura eminente en la geología española y un vulcanólogo reconocido internacionalmente. A comienzos del siglo XX participó en varias de las expediciones científicas que la Real Sociedad Española de Historia Natural organizó por la zona del protectorado español en Marruecos, que marcaron su carrera científica, principalmente en el conocimiento volcánico del territorio español tanto en África como en Canarias. Fue el primer geólogo español que pisó las islas Chafarinas. Alcanzó la cátedra de Cristalografía de la Universidad de Madrid y siempre se dedicó apasionadamente a sus tareas docentes e investigadoras hasta que una penosa enfermedad truncó su magnífico expediente.

Palabras clave: Fernández Navarro, Melilla, Chafarinas, Marruecos, vulcanología, Historia de la Geología.

Abstract: Lucas Fernández Navarro was the first Spanish volcanologist. His scientific and academic work at the Museo de Ciencias and at the Universidad Central (Madrid) led him to become an eminence in Spanish geology as well as an internationally renowned volcanologist. At the beginning of the twentieth century, he participated in several scientific expeditions which the Spanish Royal Society of Natural History organized in the Spanish protectorate in Morocco. These expeditions marked his scientific career primarily in the knowledge of Spanish territory volcanology both in Africa and in the Canary Islands. Lucas Fernández was the first Spanish geologist who set foot on the Chafarinas Islands. He became professor of Crystallography at the Universidad de Madrid where he was fully dedicated to teaching and research until his sudden death cut short his magnificent career.

Keywords: Fernández Navarro, Melilla, Chafarinas, Morocco, volcanology, History of Geology. 


\section{Introducción}

Desde que las potencias europeas pusieron sus ojos en la colonización del NO de África, y acordaron en la Conferencia de Algeciras de 1906 el reparto ordenado de ese territorio, España adquirió obligaciones, junto con Francia, para ejercer un protectorado en la zona de Marruecos. A Francia le correspondió el protectorado sur y a España el norte que, desde ese momento, se le conoció como Marruecos español.

Ya desde unos años antes de la Conferencia, las expediciones comerciales, científicas y empresariales de varios países europeos realizaban estudios en el área para determinar las posibilidades de negocio y alianzas con otros países en la explotación de Marruecos. Los recursos mineros eran uno de los objetivos más codiciados por los europeos. Dentro de esa política de Estado que señaló el mandato de la Conferencia de Algeciras, se enmarcaron los viajes científicos de exploración que realizaron varios naturalistas, entre los que estaba el geólogo Lucas Fernández Navarro, al territorio marroquí, a comienzos del siglo XX.

Fernández Navarro fue un geólogo de prestigio que supo aprovechar el vacío científico que padecía España a comienzos del siglo XX [1]. Hombre entusiasta, trabajador y honrado, se supo granjear el respeto de la comunidad científica española e internacional. Recientemente se ha publicado una pequeña biografía de él ${ }^{1}$ pero sin profundizar en las investigaciones que realizó en África, particularmente en la zona del protectorado español oriental: Melilla y Chafarinas.

\section{Infancia y juventud}

Fernández Navarro nació un frío día de enero de 1869 en Guadalajara donde su padre, Inocente Fernández-Abas, natural de Madrid, era catedrático de Matemáticas del Instituto provincial de Guadalajara (hoy IES Brianda de Mendoza). Estudió en dicho instituto desde el curso 1878 al 1883 con

${ }^{1}$ BARRERA, José Luis. "Lucas Fernández Navarro. El iniciador de la vulcanología en España”. Madrid, Congreso de septiembre de 2014. Ciencia y Técnica entre la Paz y la Guerra. 1714, 1814, 1914 / Francisco A. González Redondo (coord.). SEHCYT, (1915), vol. 2, pp. 955-962. 
buen aprovechamiento y figurando cuatro cursos en el Cuadro de Honor ${ }^{2}$. Con 15 años obtiene el título de Bachiller en 1884.

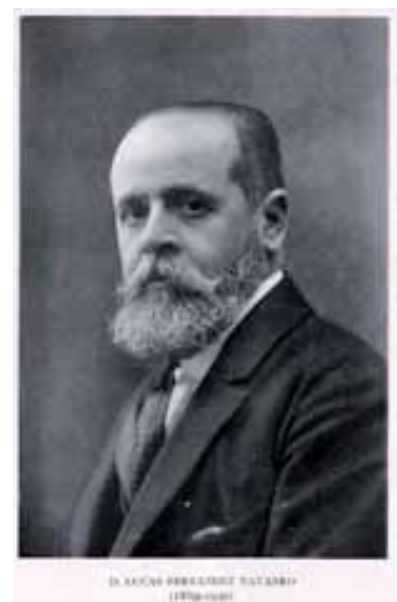

Fig. 1. Fotografía de Lucas Fernández Navarro.

De su padre heredó su afición por las Ciencias y, así, con esa vocación se trasladó a Madrid para estudiar Ciencias (sección de Naturales) en la Universidad Central. Siendo aun estudiante, ya acompañaba a su profesor Francisco Quiroga ${ }^{3}$ (mineralogista y cristalógrafo) a recoger minerales en las proximidades de Madrid ${ }^{4}$, lo que supuso el embrión de lo que más tarde llevó a Fernández Navarro a entrar como ayudante de Quiroga en el Museo de Ciencias Naturales en el año 1890.

En el pequeño espacio que dejaron al Museo compartiendo el edificio de la Real Academia de Bellas Artes de San Fernando [2], en la calle Alcalá no 13, Fernández Navarro permaneció hasta 1897. Era una persona muy laboriosa y ayudó a ordenar las colecciones mineralógicas.

\footnotetext{
${ }^{2}$ Memorias académicas. Archivo del IES Brianda de Mendoza. Guadalajara.

${ }^{3}$ Mineralogista y petrógrafo, Quiroga era de ideología krausista, liberal, miembro de la Institución Libre de Enseñanza y ayudante de Miguel Maisterra en la cátedra de Ampliación de Mineralogía, en la Facultad y en el Museo de Ciencias Naturales.

${ }^{4}$ BARREIRO, Agustín Jesus (1992). El Museo Nacional de Ciencias Naturales (1771-1935). Ed: DOCE CALLES. Colección de Historia Natural. 509 p.
} 


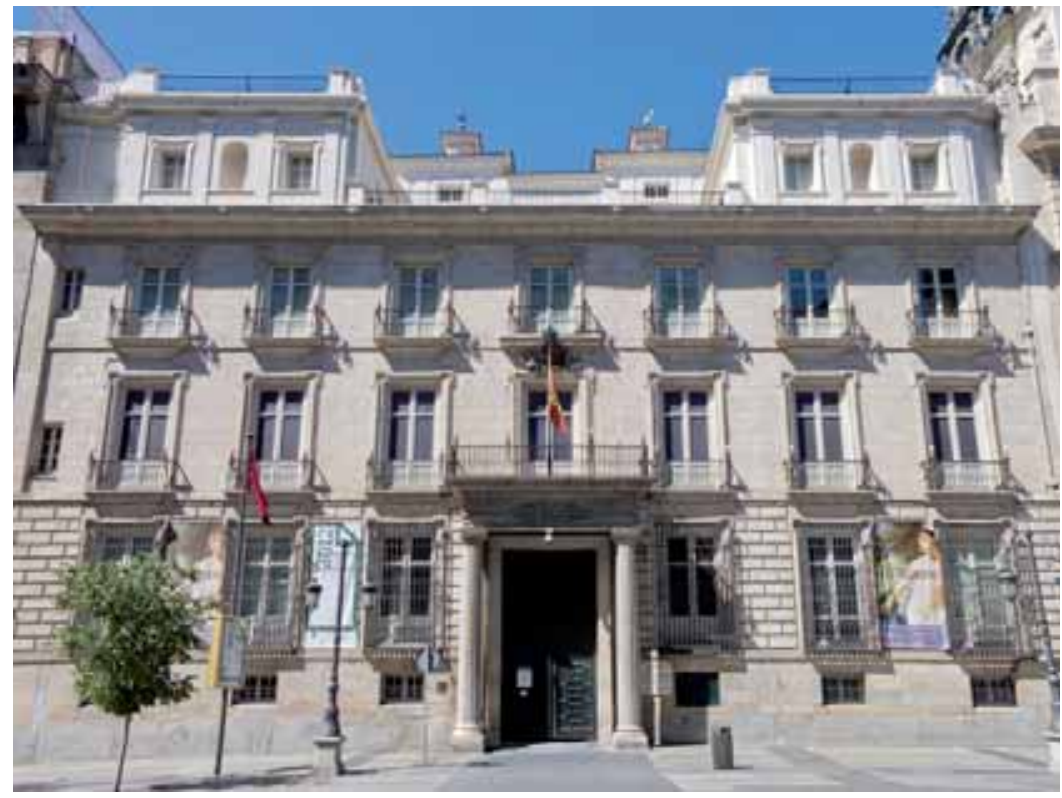

Fig. 2. Fotografía del palacio de Goyeneche, hoy Academia de Bellas Artes de San Fernando. En la parte superior del edificio estaba el local de la SEHN.

Con 21 años cumplidos, es admitido como socio en la $\mathrm{SEHN}^{5}$, la decana entre las sociedades científicas de España. Desde su fundación, la SEHN tuvo entre sus miembros a varios personajes de la nobleza que, en muchos momentos de crisis económica, sirvieron de puente entre los científicos y las autoridades del gobierno para conseguir financiación. En aquella época, la Universidad y la SEHN eran casi las únicas instituciones (sobre todo la última) que programaban expediciones científicas para el estudio del territorio, tanto en sus aspectos geológicos como biológicos.

La primera actividad de Fernández Navarro apoyada por el Museo fue la estancia de pensionado en la "Estación marítima de Zoología y Botánica experimentales" de Santander, la primera en España dedicada a la biología del mar, para desarrollar un curso de zoología marina.

${ }^{5}$ Desde 1903 pasó a denominarse Real Sociedad Española de Historia Natural, RSEHN. 


\section{Comienzos de su carrera investigadora}

Fernández Navarro se licenció en Ciencias (sección de Naturales) en julio de 1891, en la Universidad Central y, a propuesta del Museo de Ciencias, es nombrado ese año becario para el primer año en el Museo. Publica su primer artículo sobre la preparación microscópica de 50 rocas del Guadarrama ${ }^{6}$. Era un consumado petrógrafo que siguió los pasos de su maestro el geólogo gaditano José Macpherson (1839-1902).

Dos años más tarde, en 1893, defiende su tesis doctoral titulada Terrenos arcaicos de la provincia de Guadalajara, un terreno que conocía bien por ser paisaje de su infancia y juventud cuando residía con sus padres en Guadalajara. En el tribunal estaba de presidente Antonio Machado (padre) ${ }^{7}$, y Francisco Quiroga, su maestro, era uno de los vocales. Obtiene la calificación de sobresaliente. A pesar del entusiasmo con que trabajaba, la labor científica era limitada y tan lánguida como la Universidad Central ${ }^{8}$. No había prácticamente dinero para investigar y lo único que podía hacer era estudiar las muestras de minerales que había en el Museo y realizar pequeñas excursiones por los alrededores de Madrid para tomar nuevos ejemplares mineralógicos.

El año 1894 fue un mal año para él. A causa de unas fiebres tifoideas, muere a mediados de mayo su maestro, tutor y amigo Francisco Quiroga ${ }^{9}$. El departamento de mineralogía del Museo se queda sin su director y Fernández Navarro sin su guía profesional.

${ }^{5}$ Desde 1903 pasó a denominarse Real Sociedad Española de Historia Natural, RSEHN.

${ }^{6}$ FERNÁNDEZ NAVARRO, Lucas (1891). "Nota sobre 50 rocas de la Sierra del Guadarrama preparadas para su observación microscópica”. Actas de la S.E.H.N. T. XX, pp 100-103.

${ }^{7}$ Antonio Machado era muy amigo del geólogo gaditano José Macpherson que le ayudó en las tareas de geología cuando Machado estaba en la Universidad de Sevilla, y en la que llegó a ser rector.

${ }^{8}$ La Universidad Central era una institución académica que surgió en 1822 como una integración en una única institución de las enseñanzas de los Reales Estudios de San Isidro, el Real Museo de Ciencias Naturales de Madrid y la Universidad de Alcalá; es decir, pertenecer al Museo suponía estar también en la Universidad.

${ }^{9}$ BARRERA, José Luis. "El institucionista Francisco Quiroga y Rodríguez (1853-1894), primer catedrático de Cristalografía de Europa”. Boletín de la Institución Libre de Enseñanza (BILE). II época, febrero 2001, vol. 41-42, pp 99-116. Madrid. 
$\mathrm{Al}$ año siguiente, un nuevo acontecimiento negativo impacta en el Museo de Ciencias y, por ende, en el lugar de trabajo de Fernández Navarro. El Ministerio de Fomento, por necesidades de espacio, daba un plazo de apenas 48 horas para que el Museo abandonara su sede de la calle de Alcalá. El traslado precipitado de los fondos al Palacio de Museos y Bibliotecas (hoy Biblioteca Nacional), donde permaneció hasta 1910, causó el deterioro y pérdida de numerosos ejemplares y documentación de las colecciones y dejó durante años al Museo en una precaria situación. En el Museo tenía también la sede la Sociedad Española de Historia Natural.

\section{Comienza su carrera docente}

La caótica situación de los fondos del Museo en su nueva ubicación en el Palacio de Museos y Bibliotecas de Recoletos no era precisamente la más idónea para realizar un trabajo científico digno y profesional. Las colecciones embaladas en los sótanos sin poder ser expuestas, los medios económicos exiguos y el personal desmoralizado, no animaban a permanecer en el Centro. La desidia oficial por el futuro de la entidad, a la que había dejado casi sin presupuesto y plazas de científicos, hacía poco atractiva la permanencia en el centro.

Lucas Fernández Navarro, con 26 años, toda la vida por delante, y ante tanta adversidad, prefiere buscarse un empleo fuera del Museo. En 1897, decide presentarse a las oposiciones de Instituto de Enseñanzas Medias. Su deseo de casarse y conseguir más dinero le lleva a tomar esa decisión, que era la que habitualmente tomaban casi todos los naturalistas de la época que sentían inclinación por la docencia e investigación. En este contexto, gana por oposición la cátedra de Historia Natural del I.E.M. de Linares, a la que renuncia, la del I.E.M. de Almería, donde permaneció dos años ${ }^{10}$, para permutarla por la de Soria en 1900. A pesar del trabajo docente en el instituto siguió investigando en temas geológicos y mineralógicos.

En el verano de 1901, el prestigioso naturalista y entomólogo Ignacio

${ }^{10}$ MARTÍN CARDOSO, Gabriel (1931). “El profesor Fernández Navarro (1869-1930)”. Reseñas cientificas. RSEHN, VI, pp 4-22. 
Bolívar Urrutia, fue nombrado director del Museo ${ }^{11}$. Con su llegada, el centro vivió una etapa de renovación y de vigoroso impulso que le permitió recuperar gran parte de su antiguo prestigio. Rescata de Soria, en Comisión de Servicios, a Fernández Navarro para el arreglo de sus colecciones mineralógicas bajo la dirección del Jefe de la Sección que era Salvador Calderón, 18 años mayor que él. Al año siguiente es nombrado, por oposición, catedrático de Cristalografía de la Facultad de Ciencias (sección de Naturales) de la Universidad Central, vacante desde septiembre de 1899 por fallecimiento de Tomás Andrés; era la misma cátedra que había tenido Quiroga.

Probablemente, impuesto por la circunstancia de ser nombrado catedrático de esa disciplina, se vio en la obligación de difundir los conceptos de esta materia. En ese año de 1902, se publica, dentro de la colección de Manuales Soler, su libro de Cristalografía [3] ${ }^{12}$. Hasta ese momento, la dedicación de Fernández Navarro al estudio de la Cristalografía había sido pequeña y modesta, ya que su actividad docente e investigadora era la Mineralogía y Petrografía, como lo demuestra el hecho de que hasta ese año 1902, no había publicado nada sobre Cristalografía.

Desde su cátedra, la actividad de Fernández Navarro transcurría entre sus clases y sus excursiones a los alrededores de Madrid, pues no había presupuesto para hacer campañas científicas más lejanas. Pero la circunstancia cambió en 1903, cuando las gestiones ante la Corona del ministro de Instrucción Pública Allendesalazar, uno de los socios protectores de la SEHN, lograron el título de Real para la Sociedad, y se la declaró de utilidad pública, consignándose por primera vez en los presupuestos la subvención de 5.000 ptas. Esta inyección de dinero permitió realizar la campaña de los volcanes de Olot (realizada en el verano de 1904) para la que la RSEHN nombra una

${ }^{11}$ Ignacio Bolívar (1850-1944). Entomólogo vinculado al krausismo, fue director del Museo de Ciencias Naturales (1901-1934) y del Real Jardín Botánico de Madrid (1921-1930). Presidió la Junta para Ampliación de Estudios e Investigaciones Científicas de Madrid (1934-1939), sucediendo a Santiago Ramón y Cajal, y la Real Sociedad Española de Historia Natural. Fue miembro de la Real Academia Española (1930) y de la Real Academia de Ciencias Exactas, Físicas y Naturales (1944) y de diversas sociedades científicas de Europa y América.

${ }^{12}$ FERNÁNDEZ NAVARRO, Lucas (1902). Cristalografía. Manuales Soler, XXXI. Barcelona. $235 \mathrm{pp}$. 
comisión integrada por: S. Calderón, catedrático de Mineralogía en Madrid, L. Fernández Navarro, catedrático de Cristalografía en Madrid, y a M. Cazurro, catedrático en el Instituto de Gerona, uno de los pocos geólogos que sabía algo de volcanes. Fue la primera vez que Fernández Navarro tomó contacto con terrenos volcánicos.

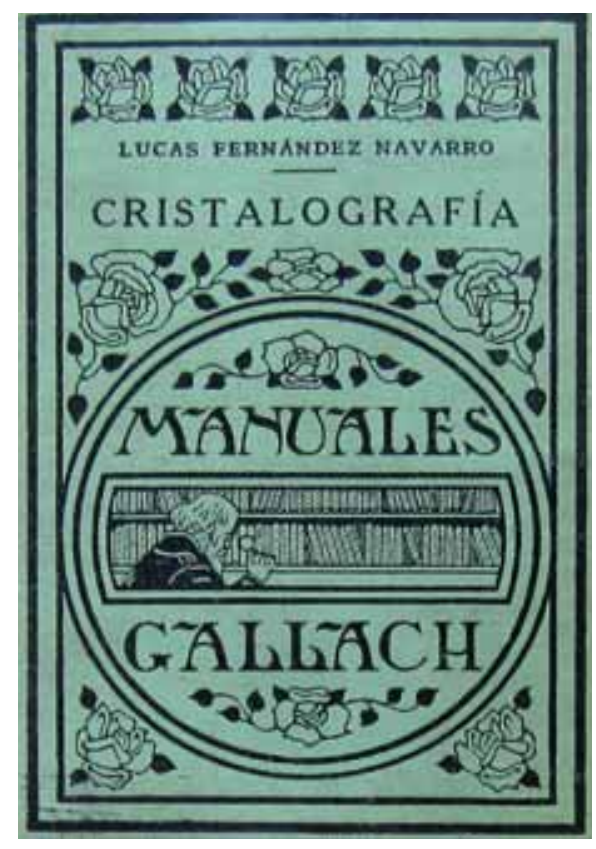

Fig. 3. Portada del libro de Cristalografía.

La campaña no pudo terminarse por la limitación presupuestaria y se decidió continuarla en el verano de 1905, aunque Fernández Navarro no fue. En esas mismas fechas viajó al norte de Marruecos comisionado por la nueva comisión que creó la RSEHN: Comisión Permanente para la exploración y estudio del Noroeste de África. Esa ocasión no había que perderla y Lucas la aprovechó intensamente.

Desde que España perdió sus colonias americanas y pacíficas, en 1898, el gobierno español buscó activamente en África una compensación a la pérdida de las posesiones de ultramar. Un nuevo imperio en África vendría a ser, desde esta perspectiva, un bálsamo para olvidar tanta catástrofe. Francia necesitaba aliados en su política de expansión africana y le ofreció a España, 
en el año 1902, el reparto de Marruecos en dos zonas de influencia. La Comisión se creó dentro de ese contexto del interés hispano-francés.

En ese mismo año de 1905, el Ministerio francés de Instrucción Pública destina, por primera vez, presupuesto para su recién fundada Misión Científica a Marruecos. No parece que sea casualidad que la creación de estas dos instituciones se produzcan al mismo tiempo y que no estuvieran acordadas en los tratados secretos que firmaron España y Francia a finales de noviembre de 1904 y comienzos de 1905. La programación de misiones de los naturalistas a Marruecos formaba parte de esa política colonial de repartirse el territorio que comenzaron ambos países. Un estudio muy completo de las misiones de los naturalistas españoles en el norte de África se tiene en el libro de Antonio Gonzalez y Alber to Gomis ${ }^{13}$.

\section{Los viajes de Fernández Navarro a Marruecos}

La Comisión estaba compuesta por personas muy relevantes de la sociedad, muy influyentes en las esferas políticas de poder, y por ilustres naturalistas [4]. Como puede verse, el secretario era Ignacio Bolívar, director del Museo de Ciencias y una persona que concitaba el respeto y consenso de todos.

El entusiasmo e interés de Fernández Navarro era tal que, doce días antes de que la RSEHN constituyera la Comisión, sale de Madrid comisionado por el Museo de Ciencias, con la intención de viajar a Melilla acompañado de otra persona. Al llegar a Málaga, el buque correo que debía llevarlos a Melilla no puede salir debido al fuerte viento. Fernández Navarro se desplaza entonces al puerto de Algeciras con la intención de cruzar a Ceuta. En una carta que envía desde Algeciras a Ignacio Bolívar, antes de embarcar, le explica el cambio de planes y le comenta lo que ha hecho “... con objeto de traer algo de África" ${ }^{14}$. A la vuelta, las cuentas que presentó el 26 de abril, fueron similares a las presupuestadas para el viaje inicial a Melilla. Según se desprende de las facturas que adjunta, estuvo también en Gibraltar las noches del 22 y 23.

\footnotetext{
${ }^{13}$ GONZÁLEZ, Antonio y GOMIS, Alberto (2001). Los Naturalistas Españoles en el África Hispana. Ed. Organismo Autónomo Parques Nacionales. Serie histórica. 425 p.

${ }^{14}$ Archivo del Museo Nacional de Ciencias Naturales (ACN), sig. 0281/007.
} 
Estaba claro que el viaje trataba de dar un golpe de efecto a la Comisión que estaba a punto de constituirse, para demostrar el interés que había por participar en la investigación y desarrollo colonial de la zona marroqui ${ }^{15}$. De esa forma accidentada comenzaba Fernández Navarro su estudio científico del norte de Marruecos. Los resultados de ese viaje no se saben cuáles fueron pues no hubo ninguna publicación referente al mismo. Tampoco se conoce la intención del viaje aunque, ante la falta de datos publicados y la inmediatez de las fechas, se puede sospechar que tenía una finalidad "propagandística” ante la inminente constitución de la Comisión Permanente para la exploración y estudio del Noroeste de África, en la que importantes personajes de la vida política y social española iban a constituir su Junta Directiva. Todo apunta a una cierta complicidad entre Bolívar y Fernández Navarro para convencer a la clase política y nobiliaria de la Comisión, de la capacidad de los naturalistas de la RSEHN para llevar a cabo empresas de esta envergadura en el norte de África.

La Comisión para el estudio del NO de África se constituyó el 1 de mayo de $1905^{16}$ con fondos del Estado y aportaciones logradas por la propia Sociedad. Aunque su intención era empezar las exploraciones en otoño, para evitar el calor sofocante del verano, la realidad fue distinta. En el mismo acto de constitución, Lucas Fernández Navarro, llevado por su impulso natural y sin querer esperar a esas fechas, ya tenía preparado su proyecto de exploración en Marruecos para los meses de junio a agosto, que había presentado a Bolívar. Tenía 36 años y un entusiasmo desbordante capaz de soslayar cualquier inclemencia climatológica o logística. También se comentó ante los asistentes, su viaje reciente a Ceuta.

${ }^{15}$ Con otras palabras, ya lo dijo Allendesalazar en el acto de constitución de la Comisión: "Dio las gracias... a todos los señores citados, por el concurso que se proponían prestar a los propósitos de la Real Sociedad que eran los de realizar una exploración científica intensiva del Noroeste de África, propósito que, aparte del grande interés científico que entrañaba, tenía también un alto fin patriótico de actualidad que no se podía desconocer, por lo que había creído conveniente solicitar desde el primer momento la protección de S.M. y el concurso de todas las clases de la sociedad y muy especialmente de la alta nobleza que por tradición bien justificada, viene unida en España a los más grandes intereses nacionales" (ACN, sig. 0284/006).

${ }^{16}$ ACN, sig. 0284/006. 


\section{COMISION PERMANENTE}

Pana LA

\section{EXPLORACIÓN Y ESTUDIO DEL NOROESTE DE AFRICA}

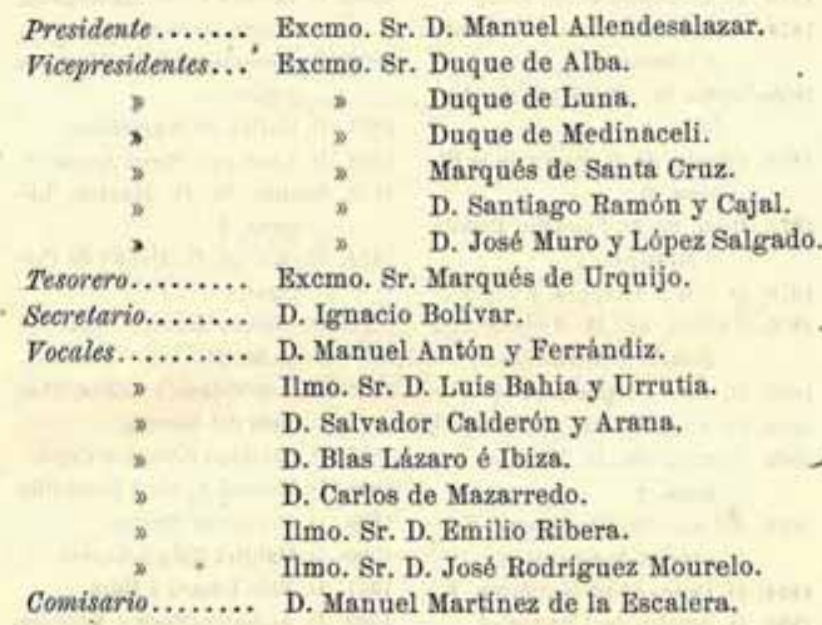

Fig. 4. Lista de los componentes de la Comisión Permanente para la Exploración y Estudio del Noroeste de África.

En el mes de mayo, y haciéndolo público en el acto de constitución de la Comisión, Fernández Navarro había presentado a Bolívar su Plan de Excursión geológica a Marruecos ${ }^{17}$, con una duración desde el día 14 de junio hasta la segunda quincena de agosto. El itinerario era: Melilla, Chafarinas, el río Muluya, volver hacia Alhucemas y Peñón de Vélez. En una segunda fase pretendía adentrarse en el Rif oriental, a Thaza para seguir hacia el oeste a Fez, Warras, Alcazarquivir, Larache o Tánger y volver a España por Cádiz. El viaje duró desde el 15 de junio hasta el 18 de agosto de 1905, con un coste total de 2.498 pesetas, y con los siguientes trayectos ${ }^{18}$ :

15 de junio: sale de Madrid con dirección a Málaga.

16-19 de junio: Estancia en Málaga.

${ }^{17}$ ACN, sig. 0281/009.

${ }^{18}$ ACN, sig. 0281/010. 
20-27 de junio: Estancia en Melilla.

27-5 de julio: Estancia en Chafarinas.

5-9 de julio: Estancia en el Peñón de Vélez.

9-12 de julio: Estancia en Alhucemas.

13-14 de julio: Estancia en Melilla.

14-22 de julio: Estancia en Alborán.

22-25 de julio: Estancia en Almería.

26 de julio a 8 de agosto: Estancia en Melilla.

9-10 de agosto: Paso por Málaga y estancia en Algeciras.

10-16 de agosto: Estancia en Ceuta.

16 de agosto: Estancia en Ronda.

En esta campaña solo se pudieron visitar las posesiones españolas por la inseguridad del resto del territorio. En la memoria publicada de los resultados del viaje reconoce que se ha limitado a describir los materiales y los paisajes ${ }^{19}$ [5]. En Chafarinas recorre las tres islas, siendo el primer geólogo español que las visitaba [6]. Destaca de ellas su interés militar por ser un lugar estratégico enfrente del río Muluya y cerca de la frontera con Argelia. Hace una descripción muy detallada de los aspectos de campo y toma muestras que luego estudia petrográficamente. Además de haberse leído la bibliografía previa que había, establece la primea cronoestratigrafía del archipielago, hace un estudio comparativo con los afloramientos volcánicos de Argelia, Alborán, Cabo de Gata y de otras zonas peninsulares. De los peñones de Alhucemas y Vélez de La Gomera afirma, muy acertadamente, que son calizas y constituyen restos aislados de la costa marroquí.

Fernández Navarro se trajo 450 minerales, rocas y fósiles que se expusieron el mes de noviembre en el Museo para que todos los socios pudieran

${ }^{19}$ FERNÁNDEZ NAVARRO, Lucas (1908). "Datos geológicos acerca de las Posesiones Españolas del Norte de África”. Mem. de la R.S.E.H.N., T.V, pp 259-340. 
observarlas. Los resultados científicos del viaje se publicaron tres años más tarde en las Memorias de la RSEHN ${ }^{20}$.

Tomo V.-Memoria 6."

DATOS GEOLÓGICOS

ACERCA DE LAS

\section{POSESIONES ESPAÑOLAS}

DEL

NORTE DE ÁFRICA

POR

L. FERNÁNDEZ NAVARRO

Catedrático de la Universidad de Madrid.

\section{INTRODUCCIÓN}

La Real Sociedad Española de Historia Naturai, en su sesión de Abril del año 1905, acord6 emprender el estudio hist6rico-natural del NW. de África. Obtenidos por suscripción los recursos necesarios para dar principio á este trabajo, y habiendo sido honrado con el encargo de contribuir á él por lo que se refiere á la Geología, me dispuse, sin pérdida de tiempo, á comenzarle en la parte que me correspondía.

Me parecí que lo primero que nos interesaba conocer eran nuestras posesiones próximas á la costa del Rif, muy poco 6 nada estudiadas en cuanto á su Geología. Este había de ser, por de pronto, el campo de mis exploraciones.

Para empezarlas, y aprovechando las vacaciones de Semana Santa, hice durante ésta mi primera excursión. No fuí en ella muy afortunado. Después de estar en Málaga varios días, esperando ińtilmente la salida del vapor que debía ir á Melilla, y viendo que el temporal que la impedía no llevaba trazas de amainar, me trasladé por tierra á Algeciras, para desde allí pa-

Memorias de la Real Sociedad españole de Hiatoria natural, tomo v, 1908. 18

Fig. 5. Primera página del artículo sobre los "Datos geológicos acerca de las Posesiones Españolas del Norte de África".

La capacidad económica de la Comisión entró en crisis a finales de 1905, y el Ministerio de la Guerra se negó a conceder a la RSEHN material de campaña para las exploraciones en África, por motivos de agitación en aquella zona ${ }^{21}$. Tampoco en la aprobación del Presupuesto del Estado iban

${ }^{20}$ FERNÁNDEZ NAVARRO, Lucas (1908). "Datos geológicos acerca de las Posesiones Españolas del Norte de África”. Mem. de la R.S.E.H.N., T.V, pp 259-340.

${ }^{21}$ ACN, sig. 0284/014/03. 
bien las cosas. Estaba previsto que en los presupuestos del año 1906, el gobierno incluyera una partida presupuestaria para los trabajos de la Comisión de la RSEHN, pero no se hizo, y hubo que presentar una enmienda al "presupuesto de las posesiones de África” para que lo incluyeran ${ }^{22}$. El tiempo corría en contra porque esto sucedía la segunda quincena de diciembre. Ignacio Bolivar, en su calidad de secretario de la Comisión, se moviliza rápidamente y contacta con varios amigos diputados (García Alix, diputado por Cartagena, y José Muro, diputado porValladolid) que le manifiestan su voluntad de apoyar su propuesta ante el Ministro de Estado. Se consiguieron 50.555 pesetas, que era algo menos de lo que solicitaban.

La amistad que tenía Bolivar con Muro debía de venir de otros ámbitos distintos a los de la Ciencia, ya que Muro era abogado y se había dedicado a la política desde hacía tiempo. Lo más probable es que su amistad estuviera relacionada con la pertenencia de ambos a la masonería. En aquella época de comienzos del siglo XX, muchos de los naturalistas e intelectuales españoles casi todos liberales- pertenecían a la masonería, y ésta ayudó mucho el desarrollo de la investigación en Ciencias Naturales. En cualquier caso, las buenas relaciones de Bolivar con la clase política, salvaron el presupuesto de la Comisión. Aquella Comisión había nacido con mucho entusiasmo y determinación pero sin fondos propios continuos para sus campañas. Esta situación, junto al voluntarismo de los socios de la RSEHN, la recordó años más tarde Fernández Navarro en unas palabras pronunciadas en su conferencia sobre Marruecos, en el Ateneo de Madrid, en 1921: “...el patriotismo de una modesta asociación particular nos salva del sonrojo de no tener activo alguno que presentar cuando se nos pregunta por nuestra labor científica actual de África” ${ }^{23}$.

Las investigaciones iniciadas en el norte de África por las instituciones españolas a comienzos del siglo XX, no estaban muy consideradas ni publicitadas por la comunidad científica europea. Igualmente, los organismos oficiales españoles tampoco apoyaban decididamente las exploraciones. Como dice textualmente en la revista Ateneo: “... No podemos creer que,

\footnotetext{
${ }^{22}$ ACN, sig. 0284/014/05.

${ }^{23}$ FERNÁNDEZ NAVARRO, Lucas (1921). Marruecos físico Valor económico del protectorado español. Conferencia pronunciada en el Ateneo de Madrid el 16 de abril de 1921.
} 
después de la Conferencia de Algeciras y del papel importante que en ella nos ha tocado representar, haya en España quien deje de interesarse por todo lo que pueda acrecentar nuestra influencia en el Noroeste africano"24.

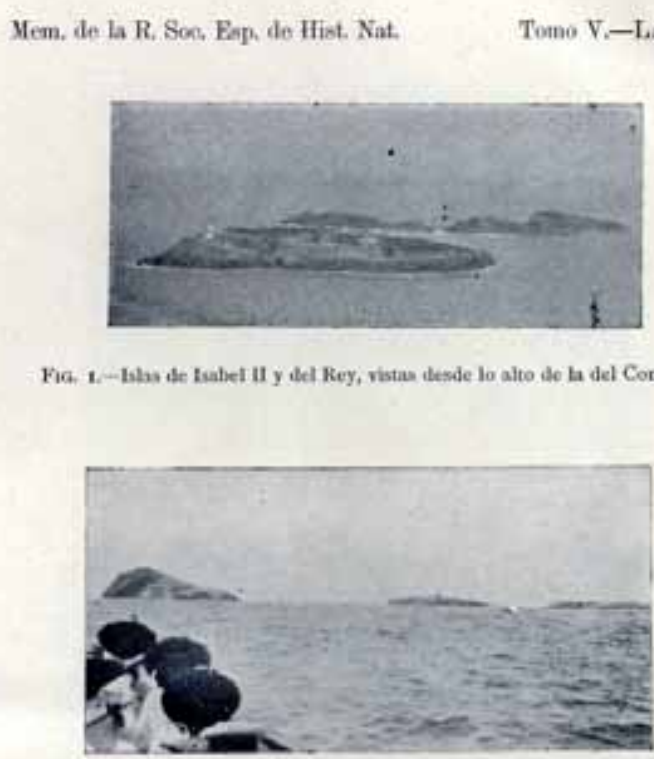

Fio 2-1 Las Chafurina desale el ST,

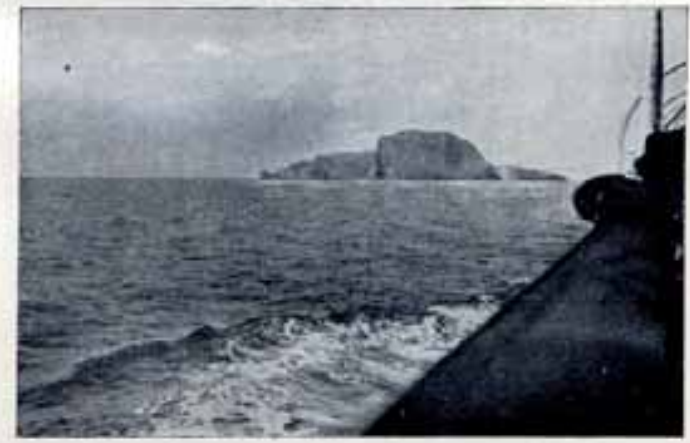

Fo. 3.-Costa eceldental de is Iala tel Coogreas,

Fig. 6. Fotografías de las islas Chafarinas tomadas por Lucas Fernández Navarro.

${ }^{24}$ FERNÁNDEZ NAVARRO, Lucas (1906). "Los naturalistas españoles en el Noroeste de África." Revista Ateneo, T1, pp 264-270. Madrid. 


\section{Fernández Navarro profundiza en el conocimiento vulcanológico}

En abril de 1906, el Vesubio tuvo una erupción muy destructiva y mató a 100 personas. Este acontecimiento le sirvió a Fernández Navarro para publicar, en la revista Ateneo de Madrid, un artículo exclusivamente bibliográfico de la dramática actualidad sobre la peligrosidad del volcán y sus erupciones históricas ${ }^{25}$. Ya tenía algunos conocimientos vulcanológicos después de sus experiencias en los volcanes de Olot, en 1904, y el viaje, al año siguiente, a la zona de Melilla e islas volcánicas de Chafarinas y Alborán. Lograría ir al Vesubio en 1912, cuando le comisionó la Junta de Ampliación de Estudios.

Por encargo de la Comisión para la exploración del Noroeste de África, Fernández Navarro va comisionado en el verano de 1906 a la isla de El Hierro para estudiar la geología que, por ser la menos conocida, interesa más estudiar. Era la primera vez que pisaba las islas Canarias; continuaba así su conocimiento de los terrenos volcánicos, que le llevaría a ser el mejor vulcanólogo que tuvo España en aquella época. El viaje lo realizó durante parte de sus vacaciones docentes de verano y, a pesar de la escasez de tiempo y dinero, también visitó Gran Canaria, Tenerife, La Palma y La Gomera ${ }^{26}$. De las observaciones que hizo, tal vez, la más llamativa es la de interpretar la depresión del Golfo como un gran cráter de explosión. En aquella época, ni Fernández Navarro ni ningún otro geólogo extranjero pensaban en fenómenos de deslizamientos como causa de esta espectacular depresión.

En julio de 1907, estalla la rebelión de las cabilas contra el Acuerdo de Algeciras de 1906 en el que las potencias europeas se repartían su influencia sobre el norte de África. La situación se puso muy tensa y un ambiente de guerra se extiende por todo el norte de Marruecos. Los continuos enfrentamientos entre los marroquíes y las tropas francesas y españolas dificultaban cualquier intento de exploración geológica.

\footnotetext{
${ }^{25}$ FERNÁNDEZ NAVARRO, Lucas (1906). “El Vesubio”. Revista Ateneo, T.I, pp 343-354 Madrid.

${ }^{26}$ FERNÁNDEZ NAVARRO, Lucas (1908). “Observaciones geológicas en la isla de El Hierro, (Canarias)”. Mem. de la R.S.E.H.N.T.V, pp 49-92.
} 


\section{El segundo viaje de Fernández Navarro a Marruecos. La campaña de 1908}

A pesar de la tensión bélica que se vivía en el norte de Marruecos, sobre todo en la zona occidental francesa, las exploraciones geológicas continuaron en la zona oriental. Así, el Museo de Ciencias, junto con la Estación Biológica Marina de Baleares, confió a Odón de Buen la dirección de una nueva campaña en Marruecos, centrada principalmente en la fauna marina. Sin embargo, se vio conveniente que también fueran geólogos para completar los estudios geológicos de la campaña anterior. Con esa finalidad, el Museo comisionó nuevamente a Fernández Navarro, no sólo porque ya había estado en el viaje anterior sino porque los conocimientos geológicos eran de un enorme interés económico por su vinculación con los recursos minerales, uno de los grandes objetivos, junto al del ferrocarril, para los capitales neocoloniales. Hay que recordar que empresarios franceses y españoles ya estaban constituyendo compañías mineras para explotar los grandes yacimientos de hierro y otras sustancias en las proximidades de Melilla ${ }^{27}$.

Tres meses duró la campaña, durante la que visitaron la zona litoral marroquí desde la frontera con Argelia hasta el cabo de Tres Forcas, incluidas las islas Chafarinas. La expedición tuvo el apoyo logístico para recorrer el territorio del general Marina, Comandante General de Melilla, y del ingeniero de las obras el puerto, Manuel Becerra, que prestó cuantos elementos tenía a su mano para montar un laboratorio portátil. Se instalaron en una fonda de la muralla de Melilla y desde allí hicieron algunas excursiones por el entorno, como la que realizaron al Gurugú, donde Fernández Navarro recogió abundantes muestras volcánicas. Otros lugares de interés geológico fueron el Monte Uixan y su yacimiento de hierro [7], desembocadura del río Muluya, el Cabo de Agua, las islas Chafarinas (donde pasaron varios días) y el cabo de Tres Forcas. Fernández Navarro estudió detenidamente los afloramientos de este último cabo y los recursos de minerales en explotación, como los de las mina Rosita, contactando con los ingenieros de minas que las

27 SAINZ VARELA, Jose Antonio y BARRERA MORATE, José Luis. “QQuiénes fueron los promotores de la Compañía Española de Minas del Rif?”. Congreso de septiembre de 2014. Ciencia y Técnica entre la Paz y la Guerra. 1714, 1814, 1914 / Francisco A. González Redondo (coord.). SEHCYT, (1915), vol. 2, pp. 971-978. 
Explotaban. Los datos obtenidos, publicados en la RSEHN ${ }^{28}$, facilitaron la confección del mapa de Marruecos hecho por el geólogo francés Gentile.

Fernández Navarro se interesó también por aspectos agrícolas y ganaderos, estudiando las necesidades hidrológicas para el desarrollo de las mismas. Sospecha que hay muchas aguas profundas (artesianas) que se podrían alumbrar para poner en regadío varias zonas del Rif, como hacían los franceses al sur de Argelia. Durante su estancia en Melilla sufrieron un terremoto, fenómeno que era recurrente en la región y que sigue siendo habitual hoy en día ${ }^{29}$.

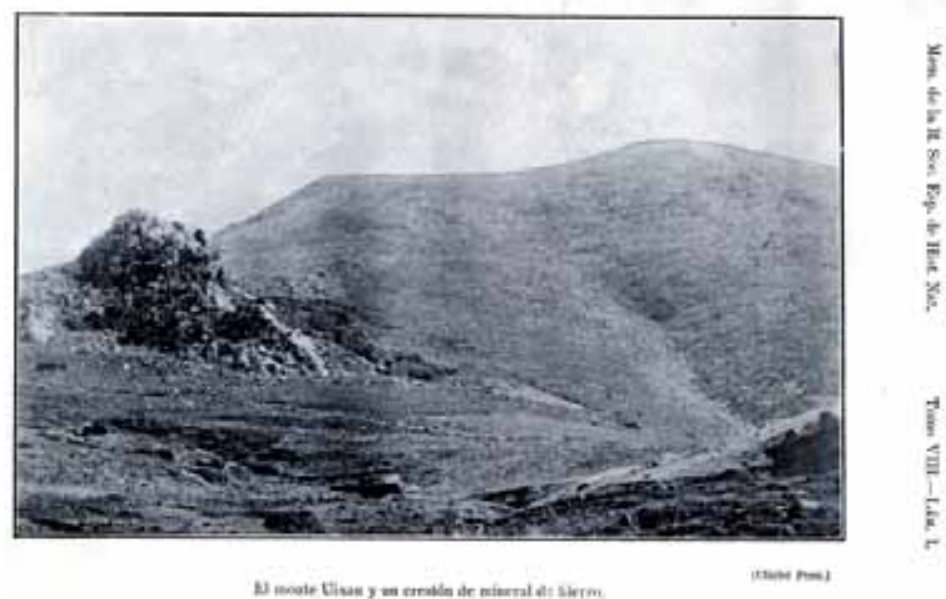

Fig. 7.Vista del crestón de hierro prácticamente puro del Monte Uixan.

\section{La erupción del Chinyero (Tenerife) y nuevos viajes y estudios vulcanológicos}

En Marruecos, la situación política de confrontación iba a peor. En julio de 1909 estalla la guerra entre rifeños y españoles en la zona oriental, que

${ }^{28}$ FERNÁNDEZ NAVARRO, Lucas (1909). "La península del Cabo de Tres Forcas (YebelGuork)”. (Noticia fisicogeológica). Bol. de la R.S.E.H.N., T. IX, 421-436. FERNÁNDEZ NAVARRO, L. (1911). Estudios geológicos del Rift oriental. Mem. de la R.S.E.H. N., T. VIII, Mem. 1, pp 5-60, 5 lám y un mapa geológico en colores.

${ }^{29}$ La sismicidad que se siente en Melilla es la que se produce de manera recurrente en el mar de Alborán, en una franja que va desde Alhucemas hasta la costa de Almería. Desde mediados de diciembre de 2015 se esta produciendo una crisis sísmica sin precedentes que se ha sentido muchas veces en la ciudad de Melilla. 
hizo imposible cualquier exploración de aquel territorio. Pero la suerte se alió con Fernández Navarro y, como consecuencia de la erupción del Chinyero, en Tenerife, en noviembre de ese mismo año, se le presenta la oportunidad fortuita de volver a Canarias. Por problemas administrativos, llego a la isla poco después de la erupción, cuando ésta había ya cesado. Por tanto, tuvo que conformarse con recoger las manifestaciones de los testigos presenciales, entre ellos su discípulo Agustín Cabrera, analizar la fase residual, cartografiar las lenguas de lava y conos piroclastos y muestrear los materiales emitidos. La memoria de la erupción se publicó en 1911 [8] y en ella se describieron perfectamente, y de manera cronológica, toda la evolución dinámica y los fenómenos de la misma ${ }^{30}$. Desde la erupción del Chinyero, Fernández Navarro se entusiasmó con el vulcanismo de Canarias, lugar donde volvería varias veces en años posteriores. De esa manera se fue haciendo una idea del fenómeno de la Atlántida y su relación con el vulcanismo, tema de su discurso de ingreso en la Academia de Ciencias Exactas, Físicas Naturales.

En 1911 es nombrado vicepresidente de la Sección de Geología en el IX Congreso Internacional de Hidrogeología, Climatología y Geología, que se iba a celebrar en Madrid en octubre de 1913. El interés de Fernández Navarro por la Hidrogeología la mantuvo toda su vida. Publicó varios artículos sobre este tema y, en ocasiones, hizo investigaciones hidrogeológicas específicas por encargo del Ministerio. En el verano de este año, viaja a Canarias, comisionado por la Junta de Ampliación de Estudios (JAE).

El Ministerio de Instrucción Pública le pensionó en 1911 para estudiar Petrografía y Vulcanología en Francia con Lacroix en el Museo de París, y con Duparc en Ginebra, e Italia. En ese viaje hizo excursiones a la región de Auvernia, montes Albanos, el Vesubio, Campos Flegreos, Capri, Ischia y museos y universidades de diferentes ciudades europeas ${ }^{31}$.

El 11 de julio de 1911 falleció en Madrid, Salvador Calderón, Jefe de la Sección de Mineralogía del Museo de Ciencias y catedrático de la Universidad

${ }^{30}$ FERNÁNDEZ NAVARRO, Lucas. (1911). Erupción volcánica del Chinyero (Tenerife) en noviembre de 1909. Anales de la Junta para Ampliación de Estudios e investigaciones científicas. T.V Mem.1, 99 p.

${ }^{31}$ MARTÍN CARDOSO, Gabriel. (1931). El profesor Fernández Navarro (1869-1930). Reseñas científicas. R.S.E.H.N., VI, pp 4-22. 
Central. Ante esta circunstancia, el 30 de septiembre, la Facultad de Ciencias propone que Fernández Navarro desempeñe, por acumulación, la cátedra de Mineralogía descriptiva ${ }^{32}$ [9]. Una vez fallecido Salvador Calderón, Fernández Navarro publica sus descubrimientos mineralógicos del norte de Marruecos, que venían a completar el gran tratado mineralógico del primero ${ }^{33}$.

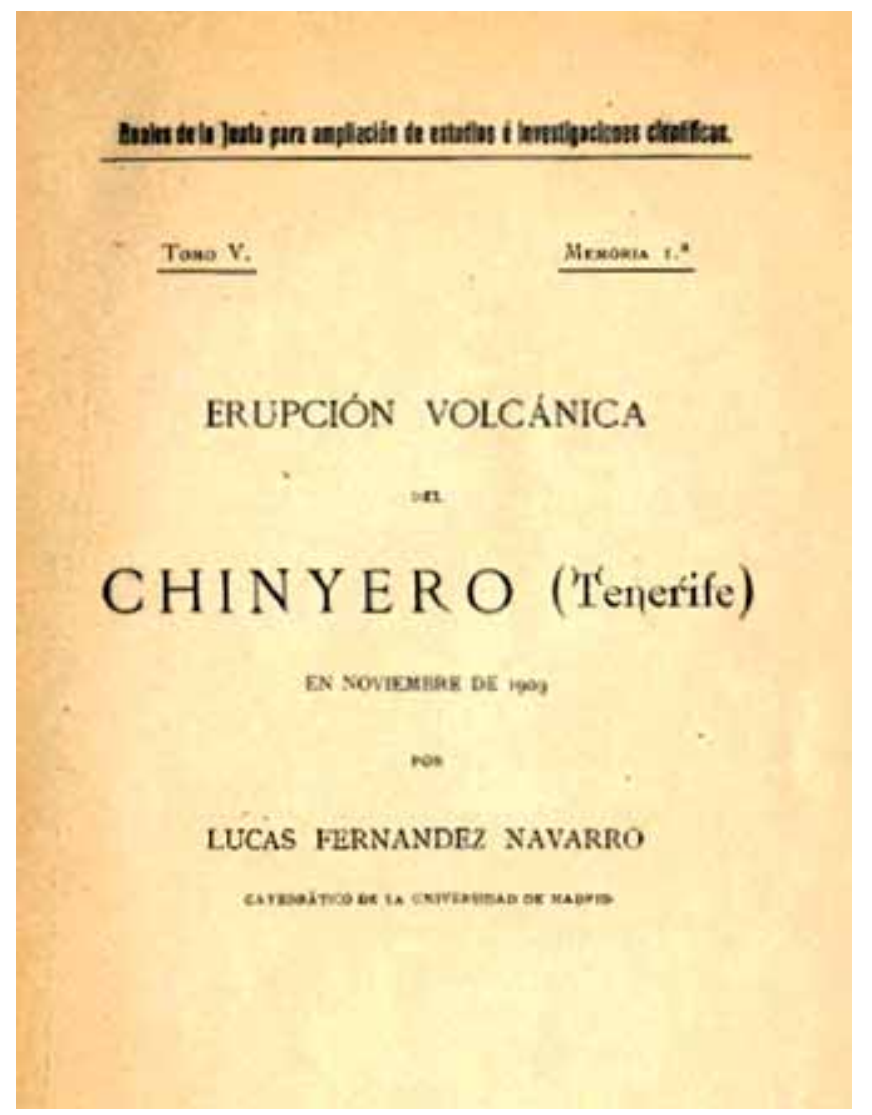

Fig. 8. Portada de la memoria de la erupción del Chinyero, en Tenerife.

${ }^{32}$ Archivo de la UCM.

${ }^{33}$ FERNÁNDEZ NAVARRO, Lucas (1912). "Minerales del Norte de Marruecos”. Bol. de la R.S.E.H.N. T. XII, pp 494-501. (En el número del 30 de julio del año 1914, lo publica también la revista Africa española, la revista de Colonización, industria, comercio, intereses morales y materiales. Pp 284-290). 


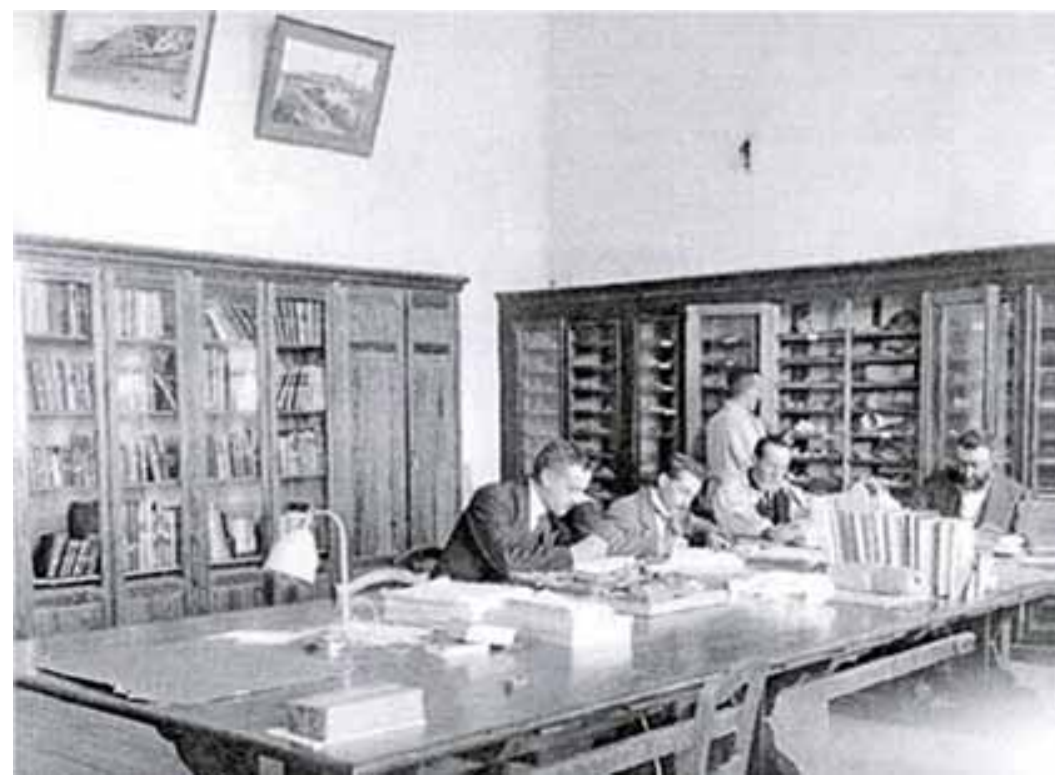

Fig. 9. Fernandez Navarro (segundo sentado por la derecha) trabajando en el Museo.

Fernández Navarro regresa a Marruecos. Viaje a Yebala y el bajo Lucus. 1913

Según los acuerdos franco-españoles firmados el 27 de noviembre de 1912, se establece el Protectorado español en Marruecos que introdujo cierta calma en el territorio marroquí. Eso permitió volver a intentar las exploraciones de los naturalistas españoles en la zona. Como escribió Eduardo Hernández-Pacheco, “...Terminadas las negociaciones y firmado el tratado internacional, es de urgencia el estudio de la zona de influencia española, pues si no, la Comisión científica que con carácter permanente acaban de organizar los franceses y que se establecerá en Rabat, la estudiara bien pronto, y no ciertamente con el fin de que aproveche a España dicho estudio"34.

La nueva Junta de la RSEHN logró del ministro de Estado, una subvención de 10.000 pesetas para la nueva exploración a la zona deYebala y el bajo

${ }^{34}$ HERNÁNDEZ-PACHECO, Eduardo et al. (1914). "Yebala y el bajo Lucus". Mem. de la R.S.E.H.N., T. VIII. 312 p. 
Lucus, que se organizó con toda rapidez. Entre los expedicionarios estaba Fernández Navarro. Los expedicionarios salieron el 9 de abril de Algeciras con destino a Ceuta. De allí se dirigieron a Alcazar, donde hicieron base para la exploración de la región yebálica. El viaje se fue prolongando y el regreso a Madrid se produjo en junio. Los resultados de las investigaciones se publicaron al año siguiente, escribiendo cada uno de los expedicionarios su correspondiente capítulo temático ${ }^{35}$.

\section{Vuelve a Canarias. Viajes al Teide}

En el verano de 1916, viaja a Canarias, comisionado por la JAE. Estudia el Teide, utilizando como base la Cañada de la Grieta $(2.150 \mathrm{~m})$ y el refugio de Altavista (3.290 m). El 29 de agosto de ese verano, es invitado a dar una conferencia en el Casino de Santa Cruz de Tenerife. Además de exponer los aspectos descriptivos del paisaje volcánico y sus formaciones geológicas, trató un aspecto que interesaba mucho a la audiencia por la repercusión en la agricultura de la isla: las aguas subterráneas. De ellas decía que para aumentarlas hay que reforestar las laderas de la isla pues en las "nubes que no llueven" esta la fuente principal de este elemento ${ }^{36}$. Vuelve al Teide 10 días al año siguiente y se instaló en el refugio de Altavista. Todos los días subía el cráter del Teide y estudiaba los gases. Como consecuencia del tremendo esfuerzo realizado, tuvo que convalecer a su vuelta a $\operatorname{Madrid}^{37}$.

\section{La última década de su vida}

En 1920, pasó a Jefe de la sección de Mineralogía del MNCN, y al año siguiente la JAE le confirió la representación en el Congreso Internacional de Hidrogeología y Geología hidrológica de Mónaco.

Estando muy cercana la celebración del XIII Congreso Geológico In-

\footnotetext{
${ }^{35}$ HERNÁNDEZ-PACHECO, Eduardo et al. (1914). Ibidem.

${ }^{36}$ FERNÁNDEZ NAVARRO, Lucas (1917). El Teide y la Geología de Canarias. (Conferencia en el casino de Santa Cruz de Tenerife). Publicada por el mismo y reproducida en Ibérica, Año IV, $\mathrm{n}^{\circ}$ 206).

${ }^{37}$ Periódico El Sol, 1 de noviembre de 1930.
} 
ternacional, que se iba a celebrar en Bruselas, el Ministerio de Instrucción Pública nombró a Fernández Navarro delegado en el Congreso, pues estaba previsto que España presentara su candidatura para que el próximo Congreso Internacional, el XIV, se celebrara en Madrid. En previsión de que la candidatura fuera aprobada, el Ministerio de Fomento ya había nombrado una "Junta Organizadora", que la presidía César Rubio, director del IGE (actual IGME), y en la que estaban como vocales Fernández Navarro y Eduardo Hernández-Pacheco ${ }^{38}$.

Desde el año 1923 al 1926 siguió viajando a Canarias, para estudiar las islas de Tenerife, Gran Canaria, Lanzarote y Fuerteventura, preparando la excursión del XIV Congreso Geológico Internacional del año 26 que se celebró en Madrid, y de la que fue miembro de la organización. Fernández Navarro se había convertido ya en un referente nacional e internacional de vulcanismo. Como prueba, baste decir que en la $2^{\text {a }}$ Asamblea de la Unión Internacional de Geodesia y Geofísica fue nombrado vicepresidente de la Sección de Vulcanología de la Unión Internacional, junto a grandes vulcanólogos y geólogos de la época, como M. A. Lacroix (Paris), H. S. Washington (Washington), A. Malladra (Observatorio Vesubiano) y G. Platania (Univ. de Catania). A su buena relación con Malladra pudo deberse la publicación del artículo sobre Canarias que le editaron en el Bull.Volcanologique ${ }^{39}$.

En 1925 tomo posesión de su puesto en la Academia de Ciencias Exactas, Físicas y Naturales, leyendo un notable discurso, en el que rechazó toda posibilidad científica de la existencia de la Atlántida.

Fernández Navarro es nombrado presidente de la RSEHN en enero de 1927, sucediendo en el cargo anual a Pío del Río-Hortega. En su pequeño discurso de presentación, Fernández Navarro destacó la atención, no exenta de cierta amargura, de que otras instituciones oficiales, y no la Sociedad, se ocuparan de los trabajos del litoral oeste africano, habiéndose abandonado

${ }^{38}$ FERNÁNDEZ NAVARRO, Lucas (1922-23). XIII Sesión del Congreso Internacional tenida en Bruselas en agosto de 1922. Ministerio de Instrucción Pública y Bellas Artes. Memoria oficial. $63 \mathrm{p}$.

${ }^{39}$ FERNÁNDEZ NAVARRO, Lucas (1924). Datos sobre el volcanismo canario. Extracto del Bull.Volcanologique, $\mathrm{n}^{\circ} 2$ (oct-dic. 1924), 29 p. Nápoles. 
las extraordinarias empresas y trabajos que se hicieron desde la Comisión Permanente para la exploración y estudio del Noroeste de África. El resultado ha sido "... el oscurantismo de nuestros trabajos, cuando realmente éramos nosotros los que habíamos traído las gallinas”.

Desde 1928, a Fernández Navarro se le declaró una enfermedad degenerativa que le afectaba al cerebro. A pesar de su enfermedad, el verano de 1930 se fue de excursión a la Sierra de Gredos. El 31 de octubre de 1930, a las seis y media de la tarde, falleció en Madrid, a consecuencia de parálisis general progresiva. Tenía 61 años. Dejó mujer (con 57 años) y tres hijos. 